\title{
Norois
}

Environnement, aménagement, société

$247 \mid 2018$

Recherche touristique : perspectives latinoaméricaines

\section{Clôtures et portails au cœur de la Caraibe continentale : une réflexion sur le tourisme, les sociétés locales et la ségrégation socio-spatiale}

Fences, gates and enclosures in the continental Caribbean: a reflection on tourism, local societies and socio-spatial segregation

Cercas y portales en el Caribe continental: une reflexión sobre el turismo, las sociedades locales y la segregación socioespacial

\section{Samuel Jouault}

\section{(2) OpenEdition}

Journals

Édition électronique

URL : https://journals.openedition.org/norois/6651

DOI : $10.4000 /$ norois. 6651

ISBN : 978-2-7535-7629-2

ISSN : 1760-8546

Éditeur

Presses universitaires de Rennes

Édition imprimée

Date de publication : 19 septembre 2018

Pagination : 77-94

ISBN : 978-2-7535-7570-7

ISSN : 0029-182X

Référence électronique

Samuel Jouault, «Clôtures et portails au cœur de la Caraïbe continentale : une réflexion sur le

tourisme, les sociétés locales et la ségrégation socio-spatiale », Norois [En ligne], 247 | 2018, mis en ligne le 19 septembre 2020, consulté le 13 janvier 2022. URL : http://journals.openedition.org/norois/ 6651 ; DOI : https://doi.org/10.4000/norois.6651 


Nö́ris

\title{
Clôtures et portails au cœur de la Caraïbe continentale : une réflexion sur le tourisme, les sociétés locales et la ségrégation socio-spatiale*
}

\author{
Fences, Gates and Enclosures in the Continental Caribbean: \\ A Reflection on Tourism, Local Societies and Socio-spatial Segregation \\ Cercas y portales en el Caribe continental: \\ une reflexión sobre el turismo, las sociedades locales y la segregación socioespacial
}

\section{Samuel Jouault}

\begin{abstract}
Faculté de Sciences Anthropologiques, université Autonome du Yucatán - Carretera a Cholul, 97000 MéRIDA Yucatán, Mexique; associé à UMR 6590 - Espaces et Sociétés, université d'Angers et au Centre d'Études Mexicaines et CentreAméricaines - CEMCA USR 337 - UMIFRE nº 16. (sam.jouault@gmail.com)
\end{abstract}

Résumé : L'espace caribéen est un espace touristique majeur où les sociétés locales sont très souvent exclues du développement touristique. Qu'ils s'agissent d'espaces littoraux ou de l'intérieur (arrière-pays), le paysage local a vu ces dernières années se dresser des clôtures et portails au cœur de villages et espaces communautaires. Si certains membres des sociétés locales ont pu s'approprier le fait touristique et développer leur propre offre, d'autres sont assujettis à des acteurs exogènes. Aussi, ce travail analyse les mises en tourisme d'espaces ruraux et littoraux et décrit des formes de ségrégation socio-spatiale dans deux villages de la Caraïbe continentale: le cas de Dzitnup au cœur de l'arrière-pays maya dans le Yucatán est mis en perspective avec le cas de Triunfo de la Cruz situé au cœur de la baie de Tela sur la côte hondurienne.

Abstract: The Caribbean is a major touristic space where local societies are often excluded from tourism development. In both coastal and inland areas, the local landscape has been changing in recent years: fences and gates are now appearing in the heart of villages and community spaces. Some members of local societies have managed to take advantage of tourism by themselves and develop tourist assets of their own, while others depend on exogenous actors. After first presenting a number of different typologies, our work goes on to study the beginnings of tourism in rural and coastal environments, and describes the forms of socio-spatial segregation observed in two villages in the continental Caribbean: the case of Dzitnup, in the heart of Yucatán's inland Maya country, is compared with that of Triunfo de la Cruz, located on Tela Bay on the Honduran coast.

Resumen: El espacio caribeño es un espacio turístico mayor donde las sociedades locales son excluidas del desarrollo turístico. Estos últimos años, el paisaje local ha visto aparecer barreras y vallas en espacios litorales o interiores, en pueblos y espacios comunitarios. Si algunos miembros de las sociedades locales han podido apropiarse del hecho turístico y desarrollar su propia oferta, otros se vuelven depen-

\footnotetext{
* Cet article a été rédigé avec l’aide du projet « Représentations littéraires de l'insularité. Vers un modèle pour la Caraïbe continentale » financé par le programme CONACYT - Sciences basiques 2016-2019. Il s'inscrit également dans le projet ECOS-Nord intitulé « Développement local et conservation: une analyse des enjeux des politiques de lutte contre la pauvreté par le tourisme alternatif dans l'État du Yucatan au Mexique », porté par l'université d'Angers et l'université Nationale Autonome du Mexique (UNAM, campus Morelia).
} 
dientes de actores exógenos. Así, esta propuesta analiza la turistificación de espacios rurales e litorales, y describe las formas de segregación socio-espacial en dos pueblos del Caribe continental: el caso de Dzitnup en la península de Yucatán esta puesto en perspectiva con el caso de Triunfo de la Cruz ubicado en la bahía de Tela en la costa hondureña.

Mots clés : Tourisme - sociétés locales - Mexique - Honduras - Mayas - Garifunas

Keywords: Tourism - local societies - socio-spatial segregation - Mexico - Mayas - Garifunas

Palabras claves: Turismo - sociedades locales - México - Honduras - Mayas - Garifunas

\section{INTRODUCTION}

Si les problématiques socio-territoriales liées au tourisme sont largement abordées en milieu urbain en Amérique Latine (García de Fuentes, 1979; Cordoba Ordoñez et al., 2001 ; López López et al., 2006; Cañada, 2013; Schwartzmann, 2013; Pérez Campuzano et al., 2014), les contributions sur ces thématiques en milieu rural sont moins nombreuses malgré le fait que le cas latino-américain regorge d'exemples divers et variés. À ce titre, la géographe Nathalie Raymond (2004 et 2005) montrait que le tourisme pouvait être un «prisme d'observation » des sociétés et présentait des travaux pionniers ${ }^{1}$ où l'étude du tourisme permet d'observer les logiques de ségrégation, très présentes en Amérique latine.

Ce texte se propose d'élargir le spectre spatial d'une série de travaux menés dans la péninsule du Yucatán au Mexique où la construction de complexes hôteliers et le développement immobilier ont été accompagnés d'un processus de restructuration territoriale dans lequel les ejidatarios ${ }^{2}$, paysans et autres pêcheurs se trouvent face à la mise en tourisme de leurs territoires (García de Fuentes et al., 2015; Marín Guardado, 2015). Bien que ces processus aient débuté sur les littoraux mexicains et dans les zones de résidences secondaires autour

1. Dans un article pour un numéro thématique de la revue Trace sur le tourisme en Amérique Latine les cas de Puerto San José et Monterrico sur la côte Pacifique au Guatemala avec un sous-titre ô combien évocateur : « la plage en Amérique Latine, chacun à sa place », traduction de «la playa en América Latina : cada quien en su lugar».

2. L'ejido est le régime juridique sous lequel s'effectue l'essentiel de la redistribution foncière mise en œuvre au vingtième siècle par l'État mexicain. À l'époque coloniale, les ejidos sont la partie des terres d'une communauté indienne, de propriété commune, qui était destinée à satisfaire les besoins en pâturages et en bois de cette communauté. L'ejido est une création des lois de la réforme agraire; ce mot désigne un ensemble de terres, y compris les terres cultivables, données en propriété par la Nation à un noyau de population agricole; l'ejido, propriété collective, est inaliénable, insaisissable, non transmissible, imprescriptible, et indivisible. Mais les terres cultivées sont divisées en parcelles individuelles (avec seulement un droit d'usage héréditaire) remises aux ejidatarios, les bénéficiaires d'une parcelle ejidale. Seuls les bois et pâtures demeurent d'usage commun. de grandes villes, ce phénomène s'est étendu de manière récente aux dites régions de refuge ${ }^{3}$ des peuples indiens d'Amérique latine (Aguirre Beltran, 1991), c'est-à-dire des espaces ruraux habités principalement par des Indiens comme c'est le cas des Mayas dans le Yucatán. L'enjeu est donc d'analyser les processus de développement touristique qui s'opèrent au travers des fermetures d'espaces littoraux et ruraux et de la délimitation de zones réservées voire exclusives destinées aux seuls touristes, dans le Yucatán, mais aussi en Amérique Centrale.

Face à l'apparition de grillages, clôtures et portails au cœur de ces espaces durant la dernière décennie, cette proposition met en perspective deux études de cas. Cette approche régionale et thématique de la Caraïbe continentale ${ }^{4}$ est une opportunité pour commencer à questionner le fait qu'il existe un modèle yucatèque comme la viabilité de transférabilité des analyses, mais aussi les liens de proximité et d'identité entre terrains d'études. La mise en perspective du cas maya avec d'autres territoires et les Garifunas au Belize, Guatemala et Honduras est justifiée par le même type de relation entre les sociétés locales et le développement touristique. Dans les deux cas présentés, le cas de l'arrièrepays maya (Dzitnup) dans le Yucatán et la baie de Tela au Honduras (Triunfo de la Cruz), les sociétés locales sont assujetties à des acteurs exogènes et perdent tout ou une partie du contrôle de l'activité touristique, tout en étant dans deux contextes différents : le premier est situé en milieu rural alors que le second est situé sur le littoral caribéen (figure 1).

Pour répondre à cet objectif, le recours au syncrétisme méthodologique inclut en tout premier lieu

3. Traduction littérale de «regiones de refugio».

4. La Caraïbe continentale, située autour de la mer des Caraïbes, du golfe du Mexique et du détroit de Floride, comprend les Guyanes (Guyane française, Guyana et Suriname), le Venezuela, la Colombie, le Costa Rica, Panama, le Nicaragua, le Honduras, le Belize, le Mexique, la Floride, la Louisiane. 


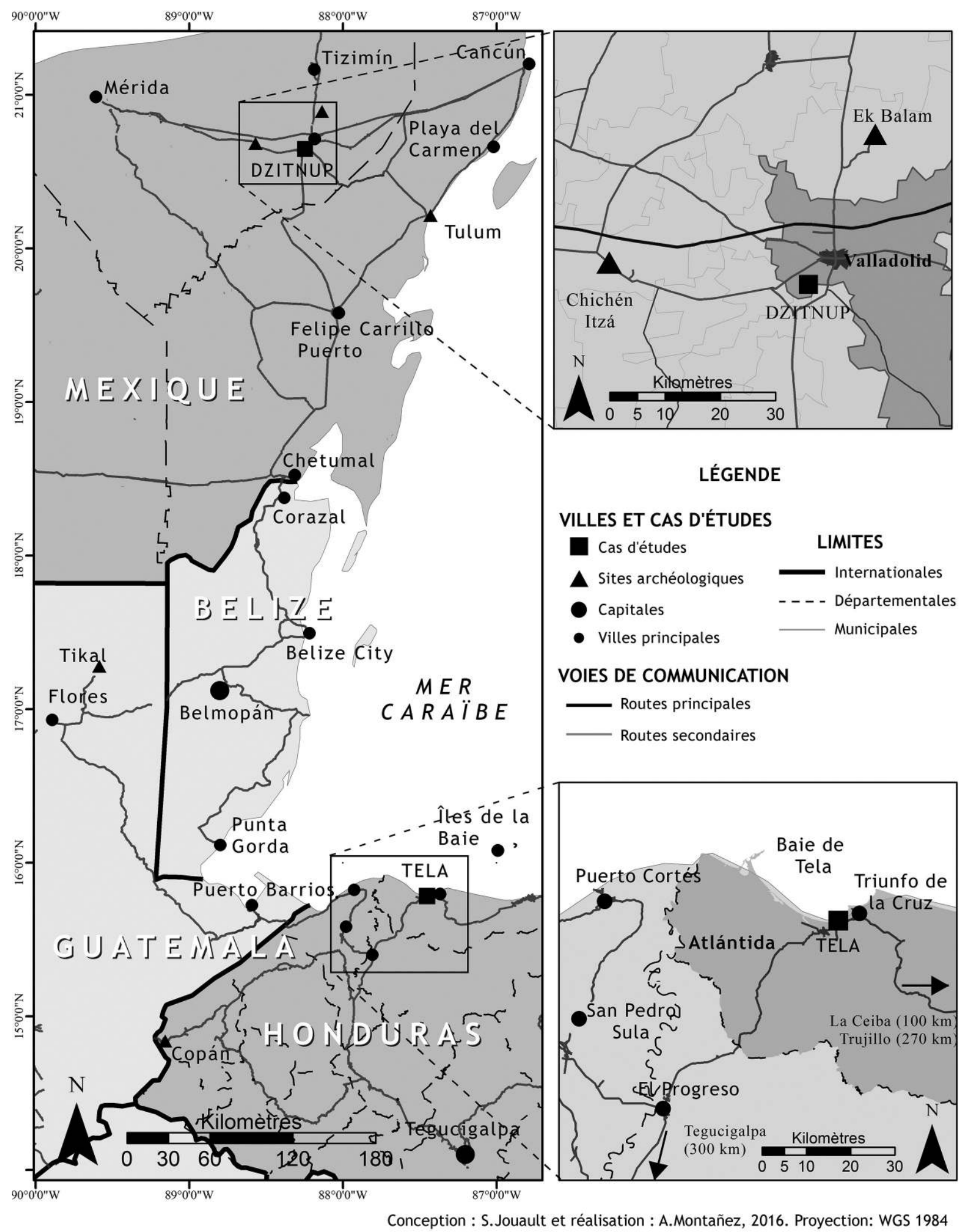

Figure 1 : Localisation des cas d'études Localization of study cases

Ubicación de los estudios de casos 
des périodes d'observation participante à Dzitnup et Triunfo de la Cruz de quelques jours à quelques semaines depuis 2006, l'accès et la consultation de documents originaux et inédits (titres de propriété collectives, contrats, etc.) dans les deux cas, la réalisation de statistiques de fréquentation des lieux touristiques et la réalisation de vingt-cinq entretiens auprès d'acteurs clés qu'ils soient villageois, acteurs endogènes et exogènes du tourisme, autorités locales et régionales. Les collectes des données ont été réalisées sur le temps-long entre 2006 et 2016. Dans la péninsule du Yucatán, entre 2012 et 2013, un questionnaire a été appliqué dans le cadre de la réalisation de l'atlas de tourisme alternatif de la péninsule du Yucatán ${ }^{5}$. Enfin, en 2016 un autre questionnaire a été appliqué dans la région.

Ainsi, dans une première partie, un bref état de l'art sur la ségrégation socio-spatiale et les discontinuités et limites spatiales précèdera une proposition de typologie du rôle des sociétés locales dans le développement touristique (seconde partie). Puis, dans les troisièmes et quatrièmes parties, les cas d'études seront décrits en présentant les mises en tourisme respectives et les situations actuelles en mettant en avant les discontinuités spatiales.

\section{BREF ÉTAT DE L'ART SUR LA QUESTION DE LA SÉGRÉGATION SOCIO-SPATIALE, LES DISCONTINUI- TÉS ET LIMITES SPATIALES}

Les géographes ont depuis longtemps observé toutes sortes de discontinuités (Brunet, 1968) et limites spatiales présentes à la surface de la terre qui influencent grandement la vie humaine (Gay, 1995 et 2016). Trente ans plus tard, le même Roger

5. Ce travail s'inscrit dans la prolongation des recherches qui ont été menées entre 2012 et 2015 et ont abouti à la publication de l'atlas de tourisme alternatif de la péninsule du Yucatán, produit qui permet aux acteurs du secteur universitaire, du secteur public et du secteur de l'économie sociale et solidaire représentés, de connaître l'importance de cette activité dans la péninsule. Il permet de cibler ses réussites, ses problématiques et ses perspectives, ainsi que la relation avec le secteur privé, le rôle des politiques publiques, et les divers types de financement en relation avec son développement et son marché entre autres aspects. Le questionnaire a été soumis à près de 200 entreprises du secteur social des trois états du Campeche, Yucatán et Quintana-Roo entre septembre 2012 et septembre 2013. Depuis septembre 2013, divers étudiants et chercheurs du Centre de recherche avancée de l'institut polytechnique national (CINVESTAV-Unité Mérida), de la faculté de sciences anthropologiques de l'université autonome du Yucatán (UADY), de l'université de Quintana-Roo (UQROO-Cozumel) et de l'université autonome de Campeche (UAC) ont constitué un comité de rédaction en parallèle du travail des géographes qui réalisent les cartes.
Brunet rappelait que «s'interroger sur les discontinuités, c'est s'interroger sur deux choses à la fois : les systèmes dans l'espace, et donc les structures ou formes spatiales» (Brunet et al., 1997). L'interrogation sur les limites et les discontinuités est fondamentale pour comprendre l'organisation de l'espace, la différenciation des lieux, la diversité de notre planète. À une échelle intermédiaire, on peut dire que le tourisme est aujourd'hui l'un des plus grands producteurs de lieux avec les innombrables stations, enclaves, communautés, paquebots qui ont été construits depuis deux siècles, ourlant les littoraux, parsemant les hautes montagnes, se propageant à travers les campagnes, voguant sur les mers, d'escale en escale (Gay, 2016). Le différentiel entre les lieux, l'altérité, est au cœur du phénomène touristique qui, en retour, est un puissant vecteur de différenciation dans l'espace.

Les discontinuités brutales étudiées ici ne sont autres que des limites mettant en contact des espaces juxtaposés. Et justement, les loisirs et le tourisme sont des producteurs d'entités spatiales. D'ailleurs, comme le rappelle Jean-Christophe Gay, « un des problèmes majeurs qui s'est posé, et qui continue de se poser dans certaines contrées, à l'aménagement touristique, est la question de l'altérité entre le touriste et le lieu visité et temporairement habité. Porteurs de leur urbanité, les touristes, aux compétences spatiales adaptatives variables, sont confrontés à un endroit nouveau, qui peut être très différent de leur territoire quotidien par la langue, les us et coutumes, les møurs, les types d'alphabet ou d'écriture, la religion, la monnaie, le niveau de vie, les normes techniques, le système de santé, etc. Au demeurant, la volonté d'aller ailleurs et de rencontrer les autres est un des fondements du tourisme. Il a donc fallu dès son essor, concevoir des dispositifs assurant la mise en relation du touriste avec des lieux et des individus différents, car une confrontation directe n'est pas sans danger » (Gay, 2016).

Ainsi, les travaux de Nathalie Raymond décrivent une ségrégation sociale et raciale en Amérique Centrale à travers le tourisme (Raymond, 2004). Cette ségrégation passe par un processus de mise à l'écart de certains groupes sociaux, résultant de stratégies spatiales concernant les lieux de résidence, mais aussi les lieux d'éducation, les lieux de travail, les lieux de loisirs, le mode de sociabilité. Cette différenciation socio-spatiale peut se faire sur des bases 
ethniques, culturelles, socio-économiques. Elle implique à la fois des mouvements subis de relégation et des mouvements choisis de l'entre-soi : un espace ségrégé peut être habité par des pauvres ou par des riches (Brunet et al., 1992; Lévy et Lussault, 2003; Géoconfluences, 2015). Si certains auteurs comme Lévy et Lussault (2003) définissent la ségrégation comme une notion liée aux analyses urbaines, d'autres travaux ont apporté un éclairage sur ses manifestations en contexte touristique à partir d'une réflexion sur les luttes pour l'appropriation de l'espace touristique, notamment dans le contexte mexicain (Marin Guardado, 2015; Marie dit Chirot, 2015). Le rôle des acteurs exogènes dans la mise en tourisme est aussi étudié par Gonzalez et Vasquez (2017) pour illustrer le développement des mégaprojets touristiques, ces projets de grande envergure (financement, emprise territoriale, retombées économiques, etc.) pensés et réalisés à l'initiative d'acteurs exogènes à la communauté.

En Amérique Centrale, les travaux d'Olivier Cuisset portent sur le village de Livingston qui joue sur sa spécificité de « ville noire » et caribéenne pour attirer des touristes en quête d'exotisme bien que la population originaire du lieu, les Garifunas, ne soient plus majoritaires à Livingston (Cuisset, 2009) ${ }^{6}$. Les Garifunas sont issus de la rencontre entre Marrons ${ }^{7}$ et Caraïbes ${ }^{8}$ sur l'île de Saint-Vincent, déportés par les Anglais, qui viennent à bout de leur résistance en 1797. Enrôlés aux côtés des « Noirs Français » dans la milice espagnole de Trujillo, qui défendait la côte des

6. Olivier Cuisset a étudié le cas de Livingston, village garifuna fondé en 1902 et situé sur la côte caribéenne guatémaltèque où les migrations successives associées à une mutation des activités économiques ont ainsi conduit à une division ethnique de l'espace. Ainsi, les Garifunas (Cuisset, 2009). La pêche artisanale a quasiment disparu face au développement de la pêche industrielle, l'agriculture de subsistance a été abandonnée, les terres vendues ou expropriées. Ce processus a été progressif; durant une bonne partie du xx siècle, les hommes ont continué à pêcher, et les femmes à cultiver la terre. L'économie traditionnelle n'a véritablement été abandonnée qu'avec les départs massifs aux États-Unis que dans la seconde moitié du $\mathrm{xx}^{\mathrm{e}}$ siècle durant laquelle les hommes d'âge moyen ont commencé à émigrer vers les États-Unis et les grands centres urbains comme New York, Los Angeles ou Miami, bientôt suivis par leurs femmes, puis par les jeunes, de telle manière qu'on puisse parler aujourd'hui d'une «migration permanente ». Quarante à soixante pourcents des hommes sont partis - la grande majorité aux États-Unis -, et la moitié des foyers environ dépendent des remesas, envois monétaires. Pourtant, Livingston a tout de même connu une croissance remarquable, au point que le tourisme y est aujourd'hui la principale activité économique (Cuisset, 2009).

7. Esclaves en fuite, lîle de Saint-Vincent a servi de refuge à plusieurs vagues d'esclaves naufragés, ainsi qu'à des fuyards des îles voisines.

8. Population amérindienne, originaire du bassin de l'Orénoque, en Amérique du Sud, les Caraïbes sont arrivés dans les petites Antilles quelques siècles avant la conquête, repoussant les Taïnos vers le nord. Le terme " garifuna » serait dérivé du mot kalinago, par lequel les Caraïbes se désignaient eux-mêmes. assauts des corsaires anglais, les Garifunas fondent des villages le long de la côte, du nord du Nicaragua jusqu'au sud du Honduras britannique (Demazière, 1994 ; Rey, 2001; Cuisset, 2009). Aujourd'hui, la culture garifuna, constituée à partir d'éléments d'origine amérindienne, africaine et européenne' se trouve au cour d'intérêts touristiques qui la dépassent, alors qu'elle est en déclin du fait de l'émigration et des bouleversements économiques et démographiques récents à Livingston (Cuisset, 2009). D’ailleurs, le même auteur montre tout au long de son travail les effets discriminants du tourisme dans ce village et comment les Garifunas sont relégués aux marges de l'espace touristique. La question géographique du centre et des périphéries renvoie aux limites et discontinuités observées sur nos terrains d'études. Sontelles des expressions illustrant les relations entre les membres des sociétés locales et le développement touristique?

\section{Sociétés LOCALES ET DÉVELOPPEMENT TOURISTIQUE : ESSAIS DE TYPOLOGIE}

Certains auteurs comme Cordero (2006) ont proposé une typologie des modèles de développement touristique opposant un modèle ségrégué à un modèle d'intégration relative. D'autres typologies seront évoquées par la suite pour caractériser la relation entre sociétés locales et développement touristique. Le premier modèle dit ségrégué est un lieu où le développement touristique est perçu comme un modèle d'enclave géré par des entreprises transnationales qui assurent la commercialisation et requiert d'importants investissements publics et privés. Les membres des sociétés locales ne sont pas pris en compte dans ce schéma de fonctionnement. Selon Cordero (2006), le cas de Cancún entre 1976 et 1984 illustrerait ce premier modèle. Le modèle d'intégration relative, lui, est une évolution du modèle d'enclave avec une intégration relative de l'économie nationale et aussi locale. En effet, ce second modèle répond à la demande touristique ou à des politiques interventionnistes en intégrant partiellement des initiatives entrepreneuriales locales. Les membres des sociétés locales se convertissent alors en prestataires de services touristiques au cœur de la destination ou sa région environnante. L'exemple de Cancún à partir de 1985 peut illus- 
trer ce second modèle. Le troisième modèle dit intégré est lui géré par des petites entreprises où prévalent les intérêts familiaux et communautaires. L'appropriation des bénéfices touristiques reste dans la propre localité et le rapport entre touristes et membres de la société locale est approfondi. L'intervention des grandes entreprises touristiques et de l'État est limitée (Cordero, 2006). Mais, le nombre d'expériences de ce troisième modèle est limité. D'ailleurs, les résultats de l'atlas de tourisme alternatif de la péninsule du Yucatán illustrent ce troisième modèle. Ainsi, en 2012, il existe 153 entreprises sociales se dédiant au tourisme alternatif au sein de leurs villages (García et al., 2015).

L'atlas de tourisme alternatif a d'ailleurs été la base d'une étude plus approfondie de ces entreprises aboutissant au « modèle régional de tourisme alternatif et économie sociale dans la péninsule de Yucatán ${ }^{9}$ » qui montre que seules $25 \%$ d'entre elles tendent au modèle d'économie sociale et solidaire (Jouault et al., 2015). Les prémices de ce modèle reposaient sur une logique de développement durable. Autrement dit à long terme elle doit pouvoir générer des revenus tout en minimisant ses impacts négatifs socio-environnementaux. Dans la péninsule du Yucatán, le tourisme est une activité saisonnière où se succèdent des saisons hautes puis basses. Nombre d'entreprises vivent selon ces fluctuations, et opèrent de manière discontinue. Le tourisme est alors un revenu qui complète les autres activités. Au cœur de l'arrière-pays maya, l'affluence touristique évaluée à environ trois millions de touristes en 2016 permet cependant aux associés de ces entreprises de vivre de l'activité touristique comme activité principale en obtenant des revenus conséquents et en les complétant avec des activités primaires (agriculture vivrière, sylviculture apiculture, etc.) mais aussi des activités telles l'artisanat ou le commerce. Les critères d'évaluation du modèle reposent d'une part sur des indicateurs de viabilité économique et d'autre part, sur des indicateurs d'économie sociale et solidaire. On identifie clairement quatre scénarios et un cinquième émergeant (tableau 1).

Au cour de l'arrière-pays maya, la question du contrôle de l'activité touristique par les membres des sociétés locale s'est rapidement posée car la tendance à la privatisation (scénario 2) décrite dans le modèle ci-dessus est omniprésente. De nombreuses entreprises sociales ont été ainsi confrontées à de fortes pressions visant la privatisation de terres ejidales. Ces privatisations revêtent différentes formes, mais le modèle omettait justement la mise en réseau avec les acteurs touristiques exogènes au village. C'est pourquoi les relations entre les membres des sociétés locales, organisées ou non, se consacrant au tourisme dans leur village et aux acteurs exo-

\begin{tabular}{|c|c|}
\hline Scénario & \multicolumn{1}{c|}{ Description } \\
\hline $\begin{array}{c}\text { Scénario 1 : } \\
\text { économie sociale et } \\
\text { solidaire }\end{array}$ & $\begin{array}{c}\text { Entreprises qui consolident simultanément des critères de viabilité économique et tendent à ceux } \\
\text { qui définissent une économie sociale et solidaire, où la distribution juste et équitable des revenus } \\
\text { générés contribue à l'amélioration des conditions de vie des associés et travailleurs. }\end{array}$ \\
\hline $\begin{array}{c}\text { Scénario 2 : } \\
\text { privatisation }\end{array}$ & $\begin{array}{c}\text { Entreprises naissant ou évoluant vers des critères opérationnels plus corporatifs que coopératifs. } \\
\text { La privatisation peut être formelle, par vente ou transformation de l'entreprise, ou couverte où se } \\
\text { maintient à convenance la figure légale de l'entreprise sans répondre à la forme organisationnelle et } \\
\text { à son fonctionnement. }\end{array}$ \\
\hline $\begin{array}{c}\text { Scénario 3: } \\
\text { paternalisme ou clien- } \\
\text { télisme }\end{array}$ & $\begin{array}{c}\text { Entreprises dont le fonctionnement est basé à long terme sur l'obtention de nouvelles subventions, } \\
\text { et pourvues d'une grande dépendance vis-à-vis des politiques gouvernementales. }\end{array}$ \\
\hline $\begin{array}{c}\text { Scénario 4: } \\
\text { échec }\end{array}$ & $\begin{array}{c}\text { Entreprises qui surgissent sous une forme d'organisation coopérative : généralement subventionnées } \\
\text { par des financements gouvernementaux, elles cessent d'opérer bien souvent pour des raisons internes. }\end{array}$ \\
\hline $\begin{array}{c}\text { Scénario 5 : } \\
\text { lentreprises familiales }\end{array}$ & $\begin{array}{c}\text { Tendance située à mi-chemin entre l'économie sociale et solidaire et la privatisation. Un des } \\
\text { exemples caractérisant ce cas (Sian Ka'an Comunity Tours à Muyil au sud de Tulum) montre un } \\
\text { certain succès du point de vue économique. }\end{array}$ \\
\hline
\end{tabular}

9. Le modèle est proposé au vu de l'hétérogénéité des résultats de l'atlas de tourisme alternatif de la péninsule du Yucatán.
Tableau 1 : Tourisme alternatif et économie sociale : cinq scénarios (Traduit et adapté de Jouault et al., 2015)

Alternative tourism and the social economy: five scenarios Turismo alternativo y economía social: cinco escenarios 


\begin{tabular}{|c|c|c|}
\hline & $\begin{array}{l}\text { Présence d'un acteur exogène asso- } \\
\text { cié à l'activité touristique }\end{array}$ & $\begin{array}{l}\text { Absence d'un acteur exogène asso- } \\
\text { cié à l'activité touristique }\end{array}$ \\
\hline $\begin{array}{c}\text { Prise de décisions collectives } \\
\text { limitée sur la gestion de l'activité } \\
\text { touristique }\end{array}$ & $\begin{array}{c}\text { Type } \mathbf{1} \\
\text { La dépendance des membres de la } \\
\text { société locale à un acteur exogène. }\end{array}$ & \multirow{2}{*}{$\begin{array}{c}\text { Type } 3 \\
\text { Le contrôle de l'activité touristique pa } \\
\text { les membres des sociétés locales. }\end{array}$} \\
\hline $\begin{array}{c}\text { Possibilité de prise de décisions } \\
\text { collectives (illimitée dans certains } \\
\text { cas) sur la gestion de l'activité tou- } \\
\text { ristique }\end{array}$ & $\begin{array}{l}\text { Type } 2 \\
\text { La contractualisation des membres des } \\
\text { sociétés locales par l'acteur exogène. }\end{array}$ & \\
\hline
\end{tabular}

Tableau 2 : Typologie du contrôle de l'activité touristique par les membres des sociétés locales (Source : Jouault, 2016) Typology of methods of controlling tourist activity used by members of local societies

Tipología de los modos de control de la actividad turística por los miembros de las sociedades locales

gènes seront au centre de la réflexion du contrôle de l'activité touristique. Deux critères ont donc été retenus pour classifier les entreprises (tableau 2) : 1) l'absence ou la présence d'un acteur exogène dans l'activité touristique portée par les membres de la société locale et à l'intérieur du village; 2) le degré de prise de décisions collectives sur la gestion de l'activité touristique, évalué selon le produit touristique commercialisé.

Si trois types se dégagent, notre intérêt porte sur le premier cas associant une prise de décisions collectives limitée de la part des membres de la société locale à la présence d'un acteur exogène (public ou privé) entraînant une dépendance des membres de la société locale à un acteur exogène associé à l'activité touristique. Le cas des cenotes du village de Dzitnup illustrera ce premier cas de figure alors que le cas de Triunfo de la Cruz montre que certains espaces du village sont assujettis à des acteurs exogènes et d'autres espaces sont restés aux mains des locaux sans pour autant présenter une organisation collective.

\section{DZITNUP, UN CAS « D'EXPROPRIA- TION » DES RESSOURCES NATURELLES COMMUNAUTAIRES?}

L'«arrière-pays maya », contraction de l'arrière-pays touristique de Cancún-Riviera maya est une région de $40000 \mathrm{~km}^{2}$ située dans la péninsule du Yucatán au Sud-Est du Mexique. Depuis les années 1970 et les débuts de la construction de la ville touristique de Cancún, puis à partir de 1990 la structuration et l'extension de la Riviera Maya, cette région caractérisée par sa population d'origine maya et une nature exubérante est marquée par un développement tou- ristique intensif. Si dans un premier temps les sites archéologiques puis les ressources naturelles ont été mis en tourisme, certains membres des sociétés locales d'origine maya ont opté ces quinze dernières années pour le développement touristique au sein de leur village (Jouault, 2016 et Jouault et al., 2017).

Le cas de Dzitnup montre tout d'abord la mainmise du gouvernement de l'État du Yucatán puis d'une entreprise privée sur les ressources naturelles du village et comment cette société locale par ses tensions internes s'est trouvée dépossédée de cette activité. Dzitnup, village situé dans la municipalité de Valladolid à quelques kilomètres de l'embranchement de la route entre Chichen Itzá et Cancún signifie « union de compagnons » en langue maya. Selon certains habitants du village, la toponymie du lieu est liée à un arbre à l'extérieur de l'église et dont les racines plongeaient dans un cenote ${ }^{10}$ au centre du village. En 2010, le village est habité par environ 1200 personnes vivant pour nombre d'entre eux dans des maisons vernaculaires avec des toits de palme ${ }^{11}$. À partir des années 1970, la ville voisine de Valladolid commence à développer des activités commerciales et touristiques et en conséquence devenir une étape sur la route Cancún-Chichen Itzá. Le tourisme devient alors une option viable de développement économique également pour les localités environnantes. Dzitnup fut une des premières localités proches de Valladolid à bénéficier de l'arrivée de touristes grâce aux deux cenotes

10. Ouverture béante typique du relief karstique du Yucatán; au fond de ce véritable puits apparaît la nappe phréatique (exemple : cenote sacré de Chichen Itzá)

11. Palme de huano, utilisée pour la couverture des toits et charpentes dans la péninsule du Yucatán. 
XKekén ${ }^{12}$ et Samulá qui s’y trouvent (Espinosa, 2013) ${ }^{13}$. Depuis 1971, il existe un accès au cenote XKekén. Dans un premier temps venaient des gens des alentours (Valladolid, Chichimila), puis la renommée du cenote s'est répandue car on entrait dans le cenote avec des torches en descendant un escalier. Les touristes étrangers ont commencé à venir dans les années 1990. À ce moment-là, il existait un parking, des toilettes et des vestiaires pour se changer, une billetterie et des locaux artisanaux tenus par les habitants de Dzitnup.

Le conflit entre deux familles, les Pootes et les Chanes, accentué par des filiations politiques antagoniques se traduira par la vente du cenote. En juillet 2003, les opposants à cette vente (87 personnes) forment une société coopérative. Le conflit s'exacerbe et se traduit par des lynchages et même des morts de telle manière que le gouvernement de l'État intervient le 20 mars 2006, en expropriant « la finca rustica X'Kekén ${ }^{14}$ » par le décret n 676. En 2007, avant les élections du Gouverneur de l'État du Yucatán, le Secrétariat de Tourisme du Yucatán formule alors un projet d'un montant d'environ cinq cent mille dollars américains ${ }^{15}$ pour réaliser des travaux. Mais celui-ci ne sera pas mis à exécution en raison des conflits qui persistent au sein du village. C'est finalement en décembre 2011 que le centre touristique de Dzitnup est inauguré et administré par CULTUR, un organisme public destiné à la gestion des espaces d'accueil des lieux patrimoniaux de l'État du Yucatán ${ }^{16}$ après l'investissement du gouvernement de l'État du Yucatán à hauteur de huit cent mille dollars incluant la construction d'un parking pour automobiles et bus, des sentiers pour mener aux cenotes, un espace administratif, des toilettes et 170 locaux commerciaux pour les artisans (figure 2

\section{- planche VIII).}

Mais de nouveau, des affrontements mettent à mal le début de la gestion puisque CULTUR prétendait louer les locaux artisanaux à une quarantaine de dollars américains mensuels ${ }^{17}$, un coût trop élevé selon les villageois. En 2012, les artisans ne

12. Kekén en langue maya signifie porc.

13. L'accès au second cenote Sambula existe depuis 2003.

14. La propriété rustique de XKeken.

15. Soit l'équivalent de six millions de pesos au taux de change officiel annuek moyende2007.

16. CULTUR gère aussi les services touristiques des sites de Chichen Itzá, Dzibilchatún, Uxmal, Ek Balam, Loltún, etc.

17. Équivalent à 460 pesos au taux de change moyen annuel USD-peso mexicain à 12,42 en 2011 . payaient ni le loyer ni l'électricité. Durant la gestion de CULTUR à Dzitnup, vingt personnes originaires de village ont été employées à la maintenance et à la surveillance du centre touristique. Mais, la gestion de CULTUR marque aussi l'édification d'une clôture autour des cenotes ségrégant l'espace communautaire de l'espace proprement dit touristique.

Les touristes, quant à eux, viennent de plus en plus nombreux découvrir les cenotes (de 68695 en 2008 à quasi 160000 en 2014) expliquant les enjeux du contrôle de ces ressources. À partir du $1^{\mathrm{er}}$ janvier 2016, les infrastructures et équipements sont administrés par une entreprise privée Toh Beh expeditions, qui essaye d'attirer des touristes nord-américains depuis Cancún. CULTUR a en effet autorisé une concession durant vingt ans à cette entreprise tout en restant l'intermédiaire entre ce dernier et les habitants du village du Dzitnup. Ainsi, le représentant du patronat CULTUR en charge du site touristique au moment du passage de témoin entre l'État et l'entreprise privée (décembre 2015) rappelle que :

«Les cenotes de Dzitnup sont actuellement loués à une entreprise en particulier, il s'agit d'une sorte d'alliance entre le gouvernement de l'Etat qui cherche à générer un développement de la zone et une amélioration des profits de telle manière à créer des emplois. Actuellement, différents investisseurs comme le propriétaire de l'hôtel Meson del Marqués de Valladolid rénove l'aire de restauration afin d'améliorer la qualité des produits et l'attention aux clients. Le patronat CULTUR sert alors d'intermédiaire et a permis la création d'emplois comme les guides, photographes [...] ou encore de manutentionnaires pour le traçage des sentiers pour les motos quad [...]. »

Nombre de conditions ont été négociées par les habitants auprès de CULTUR et du chef de l'entreprise en charge de l'exploitation des cenotes telles l'inclusion des habitants du village de Dzitnup dans le nouveau parc à thème en allouant un espace permettant la vente d'artisanat, l'amélioration de la sécurité publique en rémunérant des policiers municipaux assignés au village, la construction de toilettes publiques, la construction d'un centre de santé et l'assignation par l'Etat du Yucatán d'un médecin, l'amélioration de l'éclairage public, l'entretien des rues et trottoirs du village, ou encore l'alimentation en eau potable. Le respect de ces condi- 
tions a été au centre de tensions entre villageois et l'entrepreneur qui ont amené en août 2015 les premiers à manifester à l'entrée du parc et bloquer l'accès de celui-ci. Certaines conditions ont alors été renégociées.

Aujourd'hui, la ségrégation entre un espace dédié aux activités touristiques principalement étrangers et le reste du village de Dzitnup est indiscutable bien que le terme d'expropriation soit remis en cause par certains acteurs gouvernementaux. Le constat est éloquent puisque les ressources naturelles sont aujourd'hui clôturées (figures 2 planche VIII et figure 3). Sur l'espace autrefois communautaire, on observe une fermeture quasi totale du site touristique matérialisée par une clôture qui a été édifiée petit à petit au cours des dix dernières années. Certains locaux s'en retrouvent exclus et nombre d'entre eux proposent des services de restauration de l'autre côté des clôtures du parc thématique sur la route qui mène au centre du village (figure 3). L'infrastructure pharaonique construite pour accueillir les artisans locaux est occupée partiellement. L'argumentaire à propos de l'offre de travail aux villageois utilisé par le gouvernement et l'entreprise Toh Beh est mis à mal. En effet, peu de jeunes de Dzitnup parlent anglais couramment, ils sont alors écartés des postes de guides au profit de guides formés en anglais provenant des centres urbains et touristiques de Cancún, Playa del Carmen ou de Valladolid. Seules les tâches de photographe ou d'employés de maintenance du parc leur sont alors accessibles.

Au cours de ce conflit vieux d'une cinquantaine d'années, les forces politiques et les intérêts privés sont intervenus sans aucune prise en considération des changements et effets négatifs générés par la mise en tourisme des cenotes au sein de la société locale. La situation observée à Dzitnup rappelle aussi l'exemple d'autres conflits socio-environnementaux liés à des usages touristiques dans la région. Ainsi, Xwo et Punta Laguna, deux villages de la municipalité de Valladolid, sont actuellement le théâtre (avril 2016) de mutations spatiales liées au tourisme. Dans le premier cas, une exploitation du sous-sol (aménagement à des fins touristiques) par un consortium acquérant des terres désormais dans les espaces intérieurs de la péninsule sans obtention des permis inhérents de la part des autorités compétentes (ministère de l'Environnement et Ressources

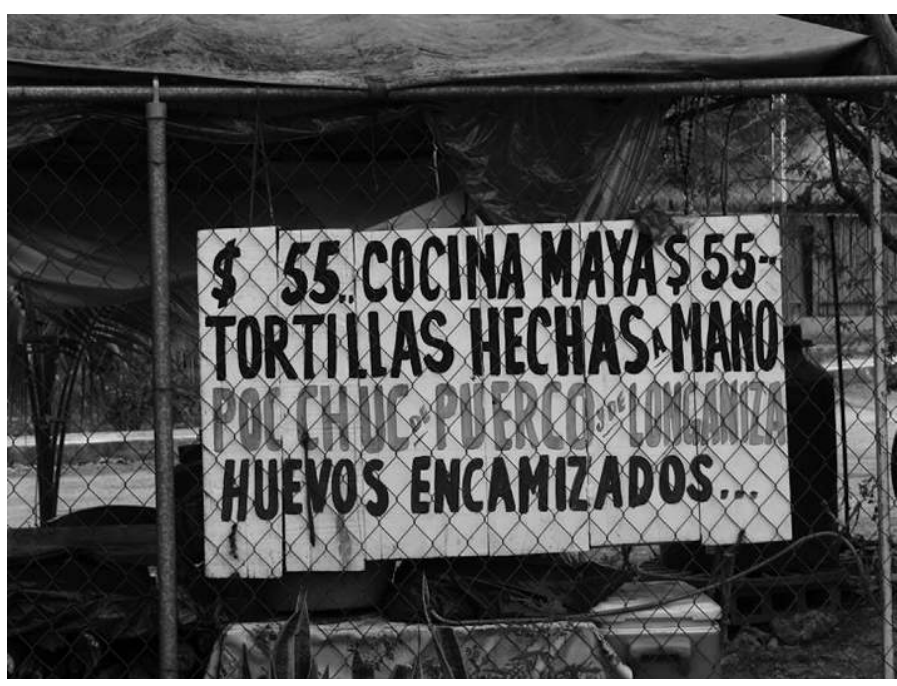

Figure 3 : De l'autre côté du grillage les locaux On the other side of the fence: local inhabitants Del otro lado de las rejas, los locales

Naturelles) a été synonyme de destruction de maisons et relogement de familles qui y vivaient. Dans le deuxième cas, le projet de parcellisation de l'ejido de Valladolid appuyée par des fonctionnaires du gouvernement de l'Etat du Yucatán afin d'y promouvoir un projet immobilier autour de la lagune oppose les membres de la société locale au gouvernement de l'État du Yucatán et ses décisions autoritaires.

Mais, face à cette pression foncière, les sociétés locales ne réagissent pas de la même manière. Ainsi, le cas de Triunfo de la Cruz montrera une certaine ségrégation de l'espace communautaire qui plus est en bord de mer associée aux résistances de certains membres de la société locale.

\section{Triunfo de la Cruz dans la baie de Tela, Honduras ou LA SÉGRÉGATION DU BORD DE MER DANS UN VILLAGE}

Le mégaprojet de la baie de Tela (figure 4) tient ses origines en 1974 sous le nom de Tornasal ${ }^{18}$ dans la municipalité du même nom entre les villages garifunas de Tornabé et Miami deux villages garifunas (figures 1 et 4). À partir des années 2000, après avoir été abandonné durant une vingtaine d'années, Indura est devenu une réalité grâce à l'appui du gou-

18. Le projet touristique s'est appelé tour à tour Tornasal, Los Micos Beach and Golf Resort et Indura. 


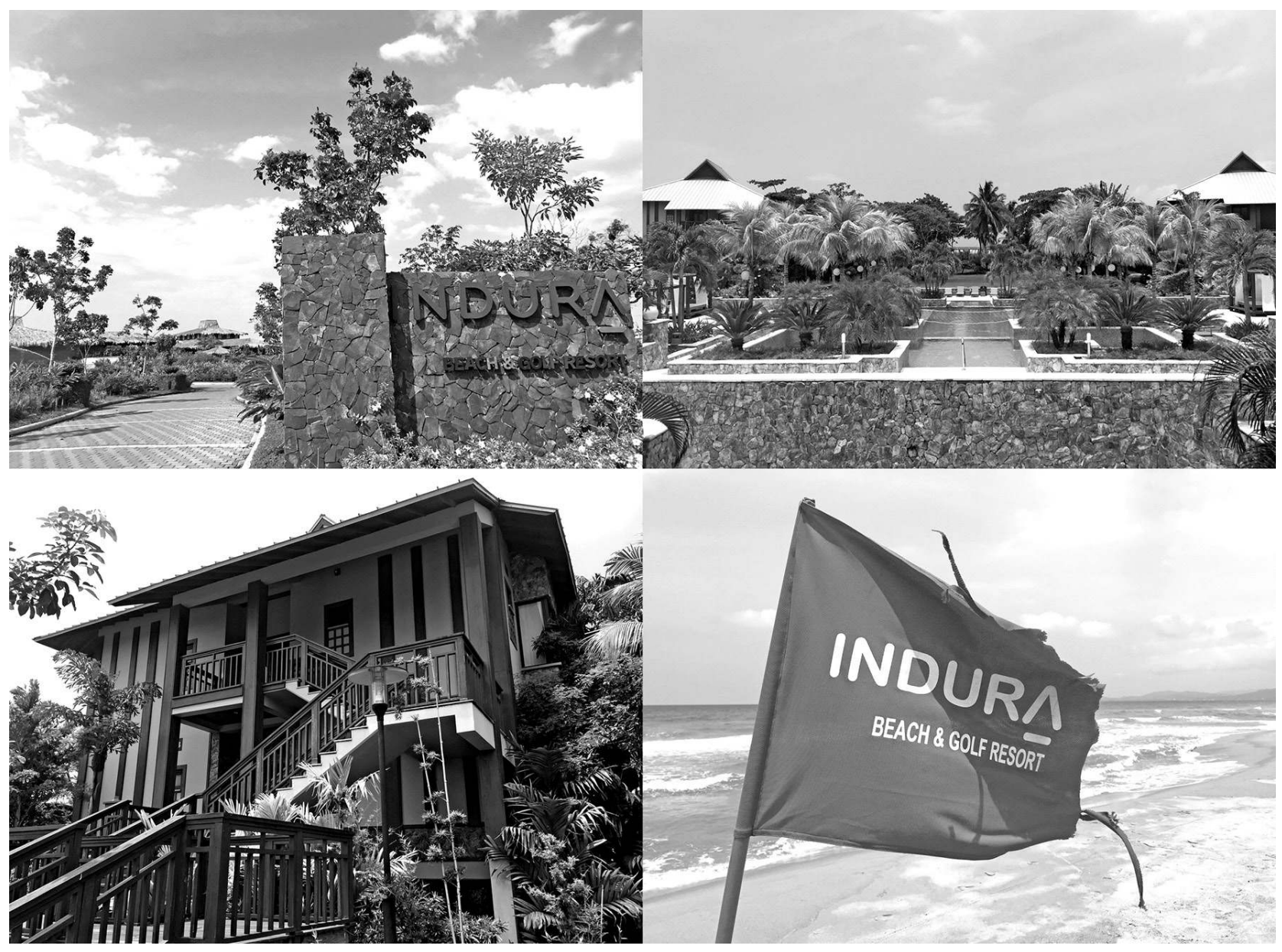

Figure 4 : Prises de vue de l'Hôtel Indura Views of the Hotel Indura El hotel Indura

vernement central hondurien et quelques groupes économiques du pays impliqués dans le projet, et ce malgré l'opposition de la population afro caribéenne de la région qui y vit depuis plus de deux siècles. Indura a été inauguré au premier trimestre de l'année 2015 après des investissements initiaux de la Banque Interaméricaine de Développement (BID), de la Banque Centraméricaine d'Intégration Économique (BCIE) pour l'infrastructure basique (voirie, électrification). La construction du complexe touristique de plus de 300 hectares dont une centaine située dans le parc national Jeannette Kawas, elle, a été gérée par la Société Anonyme Développement Touristique de la Baie de Tela (DTBT), formé par l'Institut Hondurien du Tourisme (IHT) qui possède $49 \%$ des parts et le Fonds hondurien d'investissement touristique (FITH), un conglomérat de 46 entreprises privées honduriennes qui possède $51 \%$ des actions. Dans ce montage, les villages garifunas de la Baie sont associés au projet puisque $7 \%$ des actions de l'IHT leur seront redistribuées (Trucchi, 2010). En 2012, le village de Tornabé, le plus proche d'Indura, est doté d'un système de tout à l'égout, d'une amélioration de son image urbaine et aussi de son système d'électrification.

Indura n'est pas le seul complexe touristique dans la Baie de Tela; en $2016^{19}$, on ne comptait pas moins de cinq projets immobiliers ou à vocation touristique dans la baie dont quatre étaient en fonctionnement. Le projet dit Marbella, situé à l'est du village de Triunfo de la Cruz a été stoppé par la résistance de leaders de la société locale (figure 4). En effet, de l'autre côté de la baie, Triunfo de la Cruz est une bourgade d'ap-

19. Date du dernier travail de terrain au Honduras. 
proximativement ${ }^{20} 10000$ âmes dans la municipalité de Tela. La mise en tourisme récente de la Baie de Tela a été accélérée par des acteurs exogènes et certains membres des sociétés locales y ont pris part elles aussi. En 1988, les premiers touristes étrangers sont aperçus dans le village de Triunfo de la Cruz. Un peu plus tard, en 1990, certains membres de la société locale construisent des cabanes avec des matériaux locaux coupés dans la forêt primaire de Punta Izopo pour accueillir ces touristes d'un nouveau genre. En 1992, les autorités municipales de Tela ont vendu les terres à une société-écran du nom de IDETRISA ou Inversiones y Desarrollo El Triunfo S. A. S'est alors élevée une résistance de la part d'un groupe d'habitants de Triunfo de la Cruz autoproclamé patronat pour la défense des terres du village. En effet cette vente s'est réalisée avec le consentement du chef du patronat du village $^{21}$. D'autres espaces (Playa Escondida, Tigre) ont été cédées à des familles honduriennes qui y ont construit leurs résidences secondaires fréquentées principalement durant la semaine sainte et les fins de semaine de la période estivale pour y pratiquer des sports mécaniques ou aquatiques, le tout dans une logique d'entre soi. Limpact économique est considéré comme faible (tableaux 2 et 3 ) puisque seuls quelques travailleurs originaires du village bénéficient temporellement de cette présence.

Triunfo de la Cruz, village garifuna où une grande partie de la population a migré vers les centres urbains honduriens (San Pedro Sula, Tegucigalpa, La Ceiba, etc.) est connu et apprécié à l'échelle hondurienne pour sa longue plage de sable fin bordant le Caraïbe Esmeralda. Dès 1993, des excursions locales y étaient organisées. De nos jours, ces excursions sont souvent le fait d'organisations religieuses ou associatives issues des quartiers urbains populaires de Progreso et San Pedro Sula. Ce tourisme populaire est assez significatif. En effet, durant la semaine sainte de 2015, plus de 20000 personnes ont fréquenté cette plage ${ }^{22}$. La hausse de la fré-

20. Population fluctuante due à la migration intérieure vers les centres urbains (El Progreso, San Pedro Sula, la Ceiba, Tegucigalpa) et internationale vers les États-Unis d'Amérique.

21. Les terres garifunas sont inaliénables et indivisibles, la propriété communautaire est d'ailleurs reconnue à Triunfo de la Cruz par l'obtention du titre de propriété en dominio pleno par l'État hondurien de 380 hectares en 1996 puis augmenté à 615 hectares en 2001 sur les 2840 hectares, superficie totale du village. D'ailleurs, la convention $n^{\circ} 169$ de l'Organisation Internationale du Travail a été ratifiée par le Gouvernement hondurien et protège les peuples indigènes et afrodescendants.

22. En effet, le patronat de Triunfo de la Cruz installe un péage sur la route principale d'accès au village. Les chiffres présentés dans le tableau 3. quentation durant la période estivale entraîne la reconversion d'un nombre conséquent d'habitants de Triunfo de la Cruz en auto-entrepreneurs informels proposant toutes sortes de services (vente de boissons fraîches et coco, tressage, vente de pain de coco ou de poisson grillé, etc.) (tableaux 2 et 3 ) et viennent s'ajouter à la petite dizaine de restaurants formels installés le long de la plage toute l'année.

La mise en tourisme de la baie de Tela et des villages garifunas est indissociable des politiques environnementalistes qui ont mené à la déclaration de parcs naturels dans la baie de Tela : le parc national Jeanette Kawas ${ }^{23}$, le parc national Punta Izopo et la Lencetilla gérés par la Fondation pour la Protection de Lancetilla, Punta Sal et Texiguat (PROLANSATE). Le parc Jeanette Kawas, site Ramsar ${ }^{24}$ depuis 1995 a d'ailleurs été morcelé pour la construction d'Indura, et ce avec l'appui du gestionnaire PROLANSATE. Punta Izopo, parc naturel, jouxtant Triunfo de la Cruz, a été déclaré en 1996 site Ramsar, la même année où la communauté acquiert le dominio pleno ${ }^{25}$. En 2001, Punta Izopo est déclaré parc National par l'État Hondurien. PROLANSATE en charge de la gestion du parc autorise des activités touristiques et quelques opérateurs (deux à notre connaissance) proposent des tours de découverte de la faune et la flore. Mais les retombées économiques sur le village voisin sont infimes (tableau 3).

La dualité de la mise en tourisme tant par des acteurs exogènes et endogènes conduit à une ségrégation de l'espace touristique mais aussi communautaire. En effet, les différents touristes ne se côtoient pas puisqu'il existe des barrières visibles (entre les propriétés) et invisibles sur la plage (figure 5 planche IX) qui les séparent des membres de la société locale. Pourtant dans un village garifuna, la maison et la cour font partie du système central dans l'organisation sociale de la communauté : «à chaque maison correspond un terrain divisé en plusieurs parcelles (pedazos); le pedazo souche confère le statut de chef de maison, donc la propriété foncière et le droit d'accorder ou non le droit d'usage aux autres membres du groupe familial (ou aux alliés du groupe par amitié ou union conjugale). La règle préciputaire veut que la femme transmette ce privi-

23. Du nom d'une activiste pour l'environnement originaire de Tela assassinée en 1995.

24. Convention des zones humides d'importance internationale ou Convention RAMSAR du nom de la ville où a été signée en Iran.

25. Propriété totale et/ou collective de la terre. 


\begin{tabular}{|c|c|c|c|}
\hline Lieux & Résidences secondaires & $\begin{array}{l}\text { Plage publique de } \\
\text { Triunfo de la Cruz }\end{array}$ & $\begin{array}{l}\text { Réserve naturelle } \\
\text { Punta Izopo }\end{array}$ \\
\hline $\begin{array}{l}\text { Fréquentation approxi- } \\
\text { mative }\end{array}$ & $\begin{array}{l}\text { Occupation moyenne : } \\
10 \text { personnes par maison } \\
\text { (Une cinquantaine de mai- } \\
\text { sons) } \\
\text { Max. } 500 \text { personnes }\end{array}$ & $\begin{array}{c}150 \text { bus de } 60 \text { personnes } \\
1964 \text { véhicules personnels } \\
\text { Approx. 20,000 per- } \\
\text { sonnes }\end{array}$ & $\begin{array}{c}15 \text { personnes par jour envi- } \\
\text { ron } \\
\text { Max. }=\mathbf{1 0 0} \text { personas }\end{array}$ \\
\hline Revenus approximatifs & $\begin{array}{l}\text { Entre } 30 \text { et } 50 \text { employés de } \\
\text { maison } \\
\text { (Un travailleur gagne envi- } \\
\text { ron } 4000 \text { lempiras mensuels) }\end{array}$ & $\begin{array}{l}50000 \text { lempiras soit } \\
2500 \text { USD }\end{array}$ & $\begin{array}{c}30 \text { lempiras / personnes } \\
5 \text { US / personnes }\end{array}$ \\
\hline
\end{tabular}

Tableau 3 : Fréquentation et revenus durant la semaine Sainte à Triunfo de la Cruz (2015) (Source : travail de terrain, 2016) Visitor numbers and revenue levels during Holy Week in Triunfo de la Cruz (2015)

Frecuentación turística e ingresos turísticos durante Semana Santa en Triunfo de la Cruz (2015)

lège à son fils aîné, et l'homme à sa fille benjamine, selon un principe de filiation croisé. Ce système de transmission est à la base de l'organisation traditionnelle de la vie sociale, économique (entraide) et religieuse (culte des ancêtres)»(Rey, 2001). Cette description ne mentionne aucune division de l'espace communautaire or le retour des migrants de manière temporaire ou permanente et la mise en tourisme du village déstructurent et restructurent l'espace communautaire.

Si la mise en tourisme répond à trois logiques différentes (tableau 4), elle produit inexorablement des limites dans le village qui se traduisent par une fermeture de l'espace communautaire : barrières en bois, grillages, clôtures et autres portails érigés entre les différents espaces. L'organisation de l'espace communautaire est alors fortement ségrégué en particulier en bord de mer (figure 5 - planche IX). Cette ségrégation est une réponse à la pression foncière qui sévit dans la baie de Tela depuis les années 1990. À Triunfo de la Cruz, trois zones littorales ne sont plus aux mains des locaux (Playa Escondida, une partie du quartier de Tigre et le projet Marbella entre les rivières Gama et Río Platano). Certaines familles de Tegucigalpa et San Pedro Sula ont acheté dans les années 1990 ces terres en bord de mer et ont dressé des clôtures pour séparer leurs propriétés du reste des terres de Triunfo mais aussi à l'intérieur du village. Si le tourisme et les nouveaux résidents secondaires sont à l'origine de la construction de clôtures, barrières et portails pour sécuriser leurs résidences, au cœur du village certaines clôtures sont le fait de migrants de retour des États-Unis (figure 6).

En un demi-siècle, la baie de Tela s'est peu à peu transformée d'un espace de production et logistique de la Standard Fruit Company à un espace touristique : l'offre hôtelière et résidentielle est en hausse généralement dirigée aux propres Honduriens, aux expatriés Honduriens aux ÉtatsUnis et à quelques nord-américains aventureux qui bravent les alertes de voyage du gouvernement fédéral américain. Ces mutations ont inclus les villages garifunas en marge du développement de l'économie de plantation. Mais si certains membres de la société locale sont aujourd'hui acteurs du tourisme (élites de la société locale, migrants, etc.) d'autres sont marginalisés, notamment ceux qui luttent pour faire valoir leur droit sur leurs terres accaparées par les promoteurs immobiliers et vendues bien souvent par les mêmes élites du village.

\section{Conclusion}

Le tourisme agit comme un révélateur des tensions préexistantes au sein des lieux. En effet, si la mise en tourisme d'un territoire est sujette à des problèmes et tensions au sein des sociétés locales, le fait touristique met en exergue les tensions présentes auparavant. Non seulement les deux cas mis en perspective questionnent le modèle classique de développement touristique, mais avant tout ils décrivent l'adaptation des sociétés locales face à des acteurs exogènes. Le premier cas de Dzitnup illustre l'assujettissement des membres des sociétés locales à des acteurs gouvernementaux et privés alors que le deuxième cas, celui de Triunfo de la Cruz, nuance cette dualité entre développement endogène et exogène. Et les deux études de cas illustrent cette complexe relation entre l'indigène - le local - et l'intrus - le touriste - et comment dans certains cas elle est 


\begin{tabular}{|c|c|c|c|}
\hline & Résidences secondaires & Plage «publique» & $\begin{array}{l}\text { Réserve naturelle } \\
\text { Punta Izopo }\end{array}$ \\
\hline $\begin{array}{l}\text { Type de pra- } \\
\text { tiques touristiques }\end{array}$ & $\begin{array}{l}\text { Sports aquatiques } \\
\text { «Sol y Playa » }\end{array}$ & «Sol y Playa » & $\begin{array}{l}\text { Activités à « bas impact écolo- } \\
\text { gique » et découverte de la faune et } \\
\text { la flore (observation ornithologique, } \\
\text { kayak, randonnée pédestre) }\end{array}$ \\
\hline $\begin{array}{l}\text { Modalités et } \\
\text { temporalités des } \\
\text { séjours }\end{array}$ & $\begin{array}{c}\text { Fin de semaine } \\
\text { Semaine Sainte } \\
\text { (Essentiellement en période } \\
\text { estivale) }\end{array}$ & $\begin{array}{l}\text { Excursions à la journée en } \\
\text { période estivale } \\
\text { (dimanche, Semaine Sainte) }\end{array}$ & $\begin{array}{l}\text { Excursions d'une demi-journée } \\
\text { (toute l'année) }\end{array}$ \\
\hline $\begin{array}{l}\text { Caractéristiques } \\
\text { socio-écono- } \\
\text { miques du touriste }\end{array}$ & $\begin{array}{c}\text { Classes moyennes et élevées } \\
\text { des centres urbains (San Pedro } \\
\text { Sula, Tegucigalpa) }\end{array}$ & $\begin{array}{c}\text { Classes populaires des } \\
\text { centres urbaines proches } \\
\text { (Progreso, San Pedro Sula) }\end{array}$ & $\begin{array}{l}\text { Public international et Honduriens } \\
\text { (clases moyennes, universitaires, } \\
\text { intérêt pour la nature et la culture) }\end{array}$ \\
\hline $\begin{array}{l}\text { Implication } \\
\text { des membres des } \\
\text { sociétés locales }^{\mathrm{a}}\end{array}$ & $\begin{array}{l}\text { Moyennement basse } \\
\text { (Emplois durant la construc- } \\
\text { tion et la maintenance) }\end{array}$ & $\begin{array}{c}\text { Haute } \\
\text { (Services d'alimentation - } \\
\text { économie informelle - artisa- } \\
\text { nat, pains de coco, etc.) }\end{array}$ & $\begin{array}{l}\text { Basse } \\
\text { (création de deux emplois de garde } \\
\text { forestiers et guides locaux) }\end{array}$ \\
\hline $\begin{array}{c}\text { Moteur de la } \\
\text { mise en tourisme }\end{array}$ & $\begin{array}{l}\text { Entrepreneurs privés } \\
\text { (Invernasa, etc.) }\end{array}$ & $\begin{array}{c}\text { Micro entrepreneurs } \\
\text { membres de la société locale }\end{array}$ & $\begin{array}{l}\text { Gouvernement hondurien et orga- } \\
\text { nisations non gouvernementales envi- } \\
\text { ronnementalistes }\end{array}$ \\
\hline $\begin{array}{l}\text { Impact écono- } \\
\text { mique pour les } \\
\text { membres de la } \\
\text { société locale }^{\mathrm{b}}\end{array}$ & $\begin{array}{c}\text { Bas } \\
\text { (ingrédients achetés sur le } \\
\text { lieu de résidence, salaire du } \\
\text { personnel - gardiennage, main- } \\
\text { tenance, femmes de ménage et } \\
\text { cuisinières) }\end{array}$ & $\begin{array}{c}\text { Moyennement bas } \\
\text { (achat de repas, produits } \\
\text { frais de la mer, fruits et noix } \\
\text { de cocos, boissons alcoolisés } \\
\text { et sodas) }\end{array}$ & $\begin{array}{l}\text { Bas } \\
\text { (Visite au four communautaire, } \\
\text { artisans et éventuellement un restau- } \\
\text { rant) }\end{array}$ \\
\hline
\end{tabular}

a. L'échelle de Likert utilisée ici est une technique de mesure et permet de quantifier des informations d'ordre qualitatif pour aboutir éventuellement à un classement en l'occurrence : 1. Bas, 2. Moyennement bas 3. Moyennement haute 4. Haute 5. Très haute.

b. Voir note précédente.

Tableau 4 : À chaque espace, un type de touriste (Source : travail de terrain 2010, 2012, 2015)

A specific type of tourist for each type of space

Un tipo de turista para cada espacio

modifiée puisque l'indigène se retrouve intrus sur son propre territoire (Sacareau, 1997).

La mise en perspective de ces deux cas au cœur de la Caraïbe continentale interroge aussi les pressions foncières liées aux spéculations immobilières et projets touristiques sur les sociétés locales et plus particulièrement sur la propriété collective de la terre. Initialement concentrées sur les littoraux, ces pressions foncières synonymes dans certains cas de ségrégations sont aujourd'hui observables à l'intérieur des terres. Et la description des processus revêt une importance particulière imposant à l'observateur, au chercheur ou au politicien l'impossibilité d'uniformiser cette société locale. Car loin d'être un ensemble homogène, ces sociétés locales produisent elles aussi des leaders à l'origine des ségrégations socio-spatiales et peuvent faciliter l'acquisition de terres communautaires par des acteurs exogènes.

Les luttes pour l'accès aux ressources naturelles sont au cœur de cette réflexion, tout comme la ségrégation socio-spatiale associée à la mise en tourisme de ces espaces littoraux et ruraux. Cependant on ne saurait mettre en cause seulement le fait touristique comme seul moteur de la ségrégation. Le rôle des migrants de retour des États-Unis d'Amérique peut lui aussi être évoqué. En effet, nombre d'entre eux avant de construire leur maison grâce à l'argent des remesas dressent un mur aux limites de leur terrain. La fable du XVII ${ }^{\mathrm{e}}$ siècle devenu proverbe français « Pour vivre heureux, vivons cachés » est donc d'actualité dans cette région. 


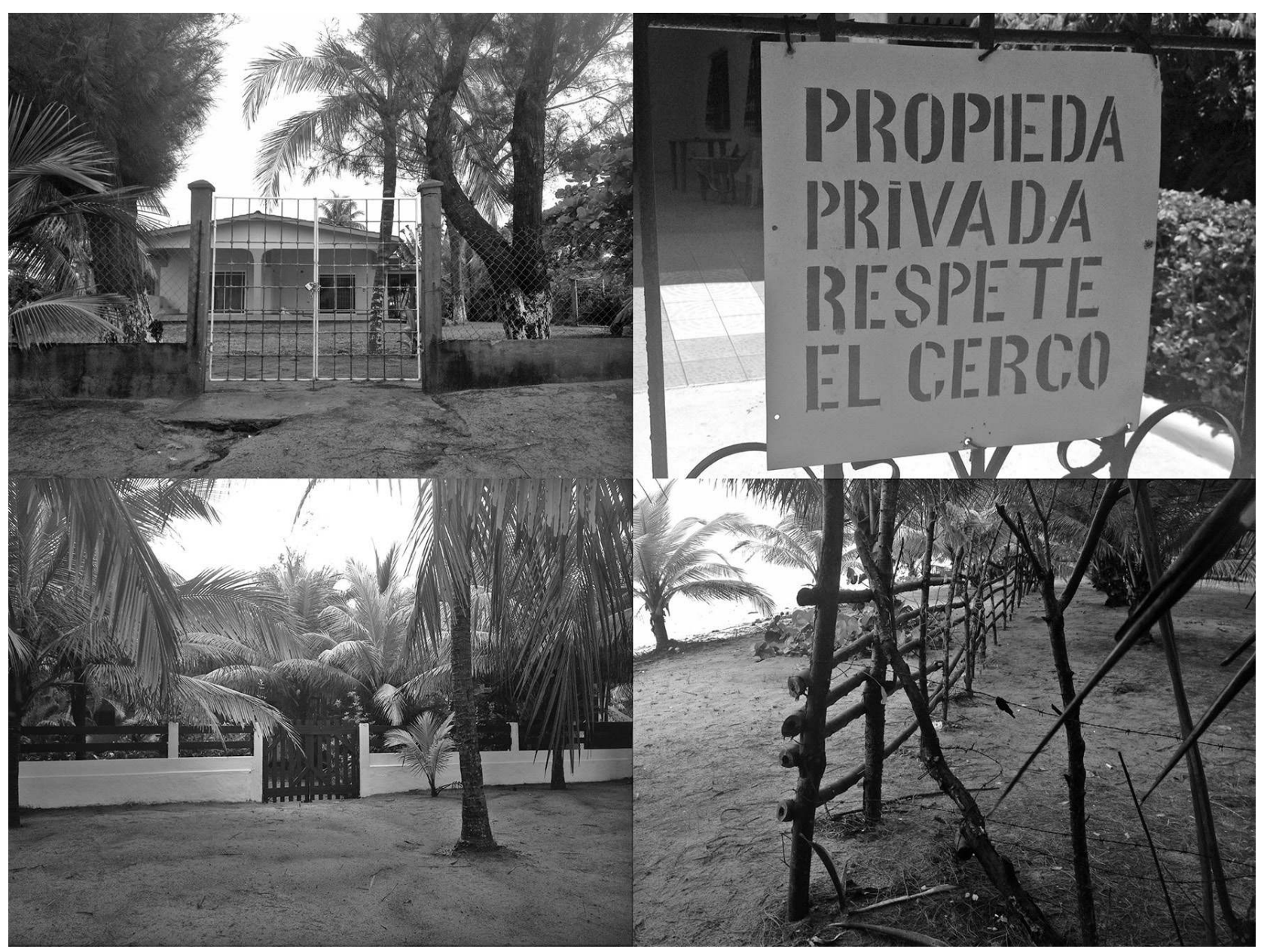

Figure 6 : Portails, grillages et clôtures à Triunfo de la Cruz Gates, fences and enclosures in Triunfo de la Cruz Portales y rejas en Triunfo de la Cruz

\section{Bibliographie}

Aguirre Beltrán, G., 1991, Obra antropológica, IX. Regiones de refugio: El desarrollo de la comunidad y el proceso dominical en Mestizoamérica, México, FCE, UV, INI, GEV, 376 p.

Brunet R., 1968, Les Phénomènes de discontinuité en géographie, CNRS, Paris, 117 p.

Brunet R., Ferras R., Théry H., 1992, Les mots de la géographie : dictionnaire critique, Paris, Reclus/La documentation Française, 470 p.

Brunet R., François J.-Ch., Grasland C., 1997, La discontinuité en géographie : origines et problèmes de recherche entretien de C. Grasland et J.-Ch. François avec R. Brunet, L'Espace Géographique, nº 4, p. 297-308.

Cañada E., 2013, Turismo en Centroamérica. Un diagnóstico para el debate, Managua, Enlace, $164 \mathrm{p}$.

Cordoba Ordoñez J., García De Fuentes A., 2001, Servidumbres del desarollo: segregación social y funcional de los espacios turísticos en Quintana-Roo (México), in $8^{\circ}$ encuentro de geografos de America Latina, p. 82-91
Cordero A., 2006, Nuevos ejes de acumulación y naturaleza. El caso del turismo, Buenos Aires, Consejo Latinoamericano de Ciencias Sociales, 211 p.

Cuisset O., 2009. Tourisme et Garifunas à Livingston, Guatemala. Economie et culture en contexte touristique. Documento de Trabajo No. 7/Document de Travail No. 7, México, Proyecto AFRODESC, 113 p.

Demazière, E. 1994. Les cultures noires d'Amérique Centrale. Paris : Karthala, 221 p.

Espinosa Hernandez E., 2013. Análisis del poder en la toma de decisiones para uso turístico de los cenotes X Kekén y Samuljá, Dzitnup, Yucatán. UADY-Mémoire de master, Mérida, 132 p.

García de Fuentes A., 1979, Turismo y subdesarrollo regional, Mexico, Universidad Nacional Autónoma de México, serie Cuadernos, 128 p.

García de Fuentes A., Jouault S., Romero, D, 2015. Atlas de turismo alternativo en la península de Yucatán. Centro de Investigación y de Estudios Avanzados del IPN-Unidad 
Mérida. Facultad de Ciencias Antropológicas-Universidad Autónoma de Yucatán. Mérida, Yucatán, 177 p.

GaY J.-Ch., 1995, Les Discontinuités spatiales, Paris, Economica, coll. " Géographie Poche », 112 p.

Gay J.-C., 2016, L'Homme et les limites, Paris, EconomicaAnthropos, $236 \mathrm{p}$.

Gonzalez, L. et Vázquez, R., 2017. Megaproyectos turísticos y ecoturísticos, del despojo al cercamiento de bienes comunes de comunidades rurales en México in Ecologia política, p. 57-61.

Jounult, S., 2016, Mayas d'hier et d'aujourd'hui. Le rôle des sociétés locales dans le développement touristique des espaces ruraux des pays émergents. Thèse de Doctorat de géographie, Université d'Angers, 693 p.

Jouault S., García De Fuentes A., Rivera Nuñez T., 2015, Modelo Regional de Turismo Alternativo y Economía Social en la Península de Yucatán, México in Otra Economía, vol. 9, n 17 , p. 164-176.

Jounult S., García de Fuentes A. y Jiménez M., 2017, L'arrière-pays touristique de Cancún Riviera Maya, in Bernard N., Blondy C., Duhamel P. (coord.). Tourisme, marges et périphéries, Rennes, Presses universitaires de Rennes, p. 233-251.

LÉvy J., Lussault M., 2003. Dictionnaire de la géographie et de l'espace des sociétés, Paris, Belin, 1034 p.

López López A., Cukier J., Sanchez Crispin A., 2006, Segregation of tourist space in Los Cabos, Mexico Journal Tourism geographies, Vol 8, 2006 - issue 4, p. 359-379.

Marie dit Chirot C., 2015, Rapports de propriété et conflits pour l'espace: approche comparative à partir des exemples de deux villes touristiques mexicaines, in Travesi C., Ponsonnet M. (dir.), Les conceptions de la propriété foncière à l'épreuve des revendications autochtones, Marseille, Les Cahiers du Credo, pacific-credo Publications, p. 253-273.

Marín Guardado G. (Coord.), 2015, Sin tierras no hay paraíso.

Turismo, organizaciones agrarias y apropiación territorial en México, El Sauzal (Islas Canarias), PASOS Revista de Turismo y Patrimonio Cultural. Colección PASOS Edita, $15,306 \mathrm{p}$.

Pérez Campuzano E., Tello, C., Everitt, J., 2014, Spatial segregation in a Tourist city: the case of Puerto Vallarta, Mexico, Journal of Latin American Geography, 13 (3), 2014, p. 87-112.

Raymond N., 2004, Los interrogantes que plantea América latina al estudio del fenómeno turístico, Trace, num. 45, p. 11-31.

Raymond N., 2005, « Le tourisme: prisme d'observation de quelques réalités latino-américaines... », L'Amérique latine, Nantes, Éditions du Temps, coll. «Questions de géographie $»$.

REy N., 2001, Les ancêtres noirs « révolutionnaires » dans la ville caribéenne aujourd'hui : l'exemple de Livingston, Guatemala. Thèse de doctorat, Université de Paris 1.

Sacareau I., 1997, Porteurs de L'Himalaya. Le trekking au Népal, Paris, Belin, 260 p.

Schwartzmann, S., 2013, Gentrification et conflits d'un quartier dans la mondialisation. Le cas de Palermo Viejo, Buenos Aires. Thèse de Doctorat de Géographie, Université de Paris 3, $419 \mathrm{p}$.

Trucchi G., 2010, Desarrollo turístico en la Bahía de Tela Los Micos Beach and Golf Resort, un proyecto polémico, Opiniones en Desarrollo. Programa Turismo Responsable. Articulo núm. 8. Alba Sud. 